INPLASY

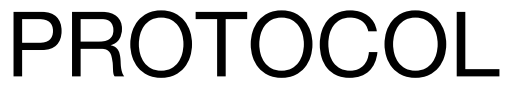

To cite: Timm et al. Movement based mindfulness therapies in patients with multiple sclerosis - a systematic review protocol. Inplasy protocol 202220102. doi:

10.37766/inplasy2022.2.0102

Received: 23 February 2022

Published: 23 February 2022

Corresponding author:

Eliane Timm

eliane.timm@unibe.ch

Author Affiliation: Institute of Complementary and Integrative Medicine, University of Bern.

Support: None.

Review Stage at time of this submission: Data analysis.

Conflicts of interest:

None declared.

\section{Movement based mindfulness therapies in patients with multiple sclerosis - a systematic review protocol}

Timm, E1; Vieregg, J2; Wolf, U³.

Review question / Objective: The aim is to review the clinical benefits of mindful moving techniques for persons with multiple sclerosis.

Condition being studied: Multiple sclerosis. Multiple sclerosis (MS) is a chronic autoimmune disease affecting the central nervous system (Gholamzad et al., 2019; Oh, Vidal-Jordana, \& Montalban, 2018). It has shown to be increasing since 2013, and as of 2020 the estimated number of people with MS is 2.8 million worldwide (Walton et al., 2020). Due accumulation of relapses or gradual progression, disability from MS is worsening over time (Cameron \& Nilsagard, 2018), which results in common symptoms like pain, imbalance, weakness, motor disorders, fatigue, depression, and more (Cameron \& Nilsagard, 2018; Guicciardi et al., 2019).

INPLASY registration number: This protocol was registered with the International Platform of Registered Systematic Review and Meta-Analysis Protocols (INPLASY) on 23 February 2022 and was last updated on 23 February 2022 (registration number INPLASY202220102).

\section{INTRODUCTION}

Review question / Objective: The aim is to review the clinical benefits of mindful moving techniques for persons with multiple sclerosis.
Condition being studied: Multiple sclerosis. Multiple sclerosis (MS) is a chronic autoimmune disease affecting the central nervous system (Gholamzad et al., 2019; Oh, Vidal-Jordana, \& Montalban, 2018). It has shown to be increasing since 2013, and as of 2020 the estimated number of people 
with MS is 2.8 million worldwide (Walton et al., 2020). Due accumulation of relapses or gradual progression, disability from MS is worsening over time (Cameron \& Nilsagard, 2018), which results in common symptoms like pain, imbalance, weakness, motor disorders, fatigue, depression, and more (Cameron \& Nilsagard, 2018; Guicciardi et al., 2019).

\section{METHODS}

Search strategy: The literature search was performed in the databases PubMed, Livivo, Embase, Web of Science, CINAHL, AMED, and Anthromedics (Merkurstab). The search was made for each intervention type separately composed of a variation of terms for multiple sclerosis, the interventions, and different terms for complementary medicine.

Participant or population: Patients with multiple sclerosis.

Intervention: Any form of Tai chi, Qi Gong, Eurythmy Therapy, Feldenkrais or Ai Chi.

Comparator: No intervention, different intervention, sham intervention, same persons over time.

Study designs to be included: All study designs were accepted, the publication had to be a peer-reviewed primary study and a full paper.

Eligibility criteria: (1) Patients with a diagnosis of multiple sclerosis. (2) At least one of the following therapies had to be applied: Tai Chi, Qi Gong, Eurythmy Therapy, Ai Chi, Feldenkrais (3) Primary studies that had investigated any kind of clinical outcome. (4) Publication had to be a peer-reviewed, full paper (5) Study had to be in either English, German or French.

Information sources: The literature search was performed in the databases PubMed, Livivo, Embase, Web of Science, CINAHL, AMED, and Anthromedics (Merkurstab). Additionally, reference lists of relevant articles were screened and the PubMed function "cited in" was consulted for those.
One google scholar internet search for all interventions was conducted at the end of the literature search, to check for the newest articles.

Main outcome(s): Any kind of clinical outcome.

Quality assessment / Risk of bias analysis: Randomized controlled trials (RCT) and controlled pre-post studies were rated using Jadad Score (Jadad et al., 1996). Non-controlled pre-post studies were rated using the "Quality Assessment Tool for Before-After (Pre-Post) Studies with No Control Group" from the National Heart, Lung, and Blood Institute (NHLBI, 2021). Case studies were rated using the CARE Checklist for Case reports (Gagnier et al., 2013). Study quality was assessed by two authors independently, discrepancies were clarified.

Strategy of data synthesis: Systematic review.

Subgroup analysis: Not applicable.

Sensitivity analysis: Not applicable.

Language: English, German, French.

Country(ies) involved: Switzerland.

Keywords: Multiple sclerosis; Tai Chi, Qi Gong, Eurythmy Therapy; Feldenkrais; Ai Chi.

Contributions of each author:

Author 1 - Eliane Timm.

Email: eliane.timm@unibe.ch

Author 2 - Julia Vieregg.

Email: julia.vieregg@unibe.ch

Author 3 - Ursula Wolf.

Email: ursula.wolf@unibe.ch 